$16^{\text {th }}$ International Congress of Metrology, 06001 (2013)

DOI: $10.1051 /$ metrology/201306001

(c) Owned by the authors, published by EDP Sciences, 2013

\title{
Terrestrial Laser Scanner (TLS) equipment calibration
}

\author{
M. T. Fernández Pareja ${ }^{1}$, J. de Vicente y Oliva $^{2}$ and A. García Pablos ${ }^{3}$ \\ Department of Surveying Engineering and Cartography.Universidad Politécnica de Madrid \\ Campus Sur. Carretera de Valencia km 7. 28031 Madrid. Spain
}

\begin{abstract}
The aim of this study is to develop the measurement procedures in order to evaluate the quality of the observations carried out using Terrestrial Laser Scanner (TLS) equipment. It tries, in addition, to estimate an uncertainty value that allows the users to know the reliability of the instrument measurements. The intention of this paper is to describe a working methodology, able to be reproducible, without the essential need of having a very complex infrastructure.
\end{abstract}

\section{Introduction}

In recent years, various measurement systems in the area of Geomatics have been developed, all of them based on the massive data acquisition in real time with high metrological quality. Newest measurement systems provide abundant spatial information and offer significant advantages in many applications. The selection of any of the systems will depend on the requirements of the project but, in all cases, such systems must be subject to metrological control that allows determining the uncertainty in measurement.

The Terrestrial Laser Scanner (TLS) equipment arises from the need to optimize the spatial data capture methods. This equipment has some advantages versus Total Stations and GNSS (Global Navigation Satellite Systems), since it allows massive data capture continuously. Laser sweepers have a large number of applications in different fields, such as industrial applications, cultural heritage documentation, auscultation of large structures, etc. The existence of these applications create the need for the reliable knowledge of the quality of the observations obtained with TLS equipment as well as the definition of a specific norm allowing users to determine the state of one particular equipment model [1].

With this research study we intend to contribute to the design of a calibration procedure that would allow assessing the quality of the observations carried out with TLS equipment. To that effect, on the one hand the factors acting on the results of measurements are analysed in order to estimate an uncertainty value associated to every point captured with a particular model

\footnotetext{
${ }^{1}$ te_fer@topografia.upm.es

2 jvo@etsii.upm.es

3 lcmigt@topografia.upm.es
}

of TLS equipment, with its 3D coordinates; on the other hand we intend to estimate an uncertainty value associated to a surface model. By reproducing different measurement conditions, the previously established methodology is applied to determine the values that would allow knowing the repeatability and reproducibility of the measurements made, all of this following the guidelines and recommendations contained in the Guide to the Expression of Uncertainty in Measurement [2]. In the present study TLS instruments from different manufacturers based on different measurement principles were used.

\section{TLS equipment}

TLS equipment is a powerful real-time measuring geomatic tool. This real-time measurement system provides abundant spatial information, the subsequent treatment of which allows geo-referencing of the captured point clouds, its representation in 3D digital format, digital elevation models, point cloud videos, orthoimages, etc. The different equipment models stand as "black boxes" [3], hence in order to make sure that the results are satisfactory, the uncertainty sources coming up during the measurement process must be detected, the contribution of each one of them should be quantified and the appropriate calibration and adjustment processes should be developed.

In this study the following TLS equipment models of different manufacturers have been employed based on different measurement principles:

- TrimbleGX3D Scanner, based on the principle of measurement by pulses or flight time, 
Leica HDS 6200, based on the principle of measurement of phase difference, and

Scan Station C10, based on the principle of measurement by pulses or flight time.

The scanned point cloud is referred to the internal Cartesian system of the browser defined by the origin coinciding with the optical centre of the instrument and by three axes forming a clockwise trihedron (main axis, collimation axis and horizontal axis of rotation). The final product may be obtained in a local reference cartographic system although in certain geomatic projects it is necessary to get it in a specific geodetic frame; this will require knowledge of the 3D position of the points from which the sweeping will take place in that reference system. After the explorations have been carried out and the point cloud has been geo-referenced, it will be necessary to filter the raw data in order to eliminate the noise and the irrelevant information.

\section{Planning of observations}

The scanned signals were three spheres measuring about $60 \mathrm{~mm}$ in diameter distributed in the space so that they form a triangle as close as equilateral as possible. The measures were carried out both under field conditions, where they were affected by meteorological circumstances at the time of observation, and under laboratory conditions where the temperature was registered and controlled during the time of observation [4]. The in-field data capture was carried out at the Escuela Técnica Superior de Ingenieros en Topografía, Geodesia y Cartografía (ETSITGC) of the Universidad Politécnica de Madrid (UPM). The measures under laboratory conditions, Figure 1, were carried out at the Laboratory of Metrologic Control of Geodetic and Topographic Instruments (LCMIGT) located within the ETSITGC.

Figure 1. Measurement performed under laboratory conditions

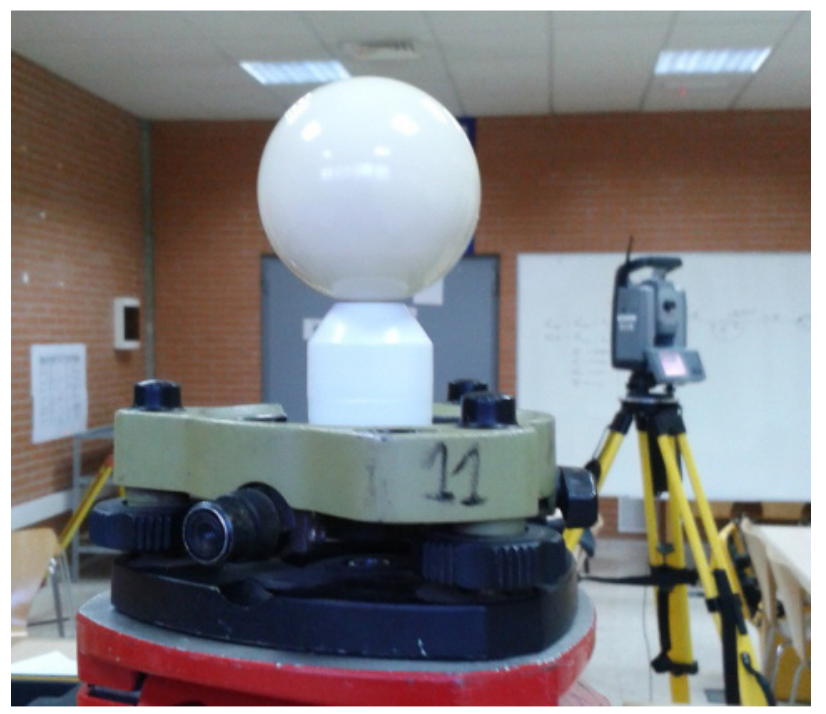

Both under field and lab conditions, three spherical signals named A, B and C were placed in the positions displayed in Figure 2, and the TLS equipment was placed in the position named Base. The position of the aiming signals was not altered during the observation.

Figure 2. Diagram of the arrangement of in-field and lab measurements
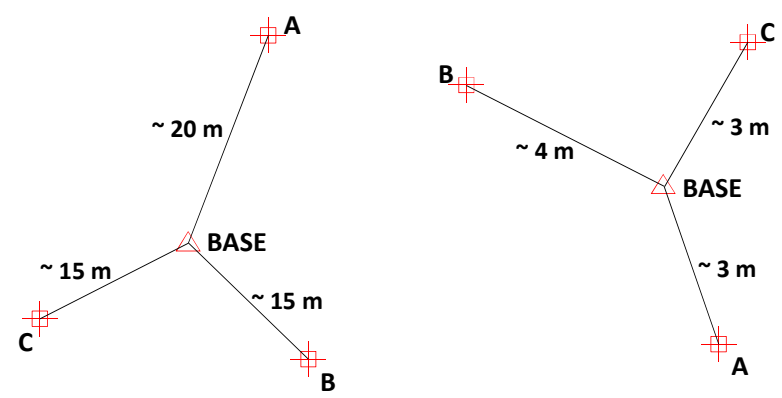

\section{Evaluation and expression of TLS equipment measuring uncertainty}

The TLS measurement equipment models, capable of sampling a surface, registering the $3 \mathrm{D}$ coordinates $(x, y, z)$ of every point making up the swept area, provide a model of the scanned surface; this provides information about shape and appearance of that surface. Consequently the definition of the modelled surface will depend on the quality of the points that make it up.

The model function of every scanned point is defined by the following expressions:

$$
\begin{aligned}
& x=r \cdot \cos \lambda \cdot \cos \varphi+C_{\text {signal }}+C_{V \varphi}+C_{C \lambda}+C_{R x}+C_{C A}+C_{a x i s} \\
& y=r \cdot \cos \lambda \cdot \sin \varphi+C_{\text {signal }}+C_{V \varphi}+C_{C \lambda}+C_{R y}+C_{C A}+C_{a x i s} \\
& z=r \cdot \sin \lambda+C_{\text {signal }}+C_{V \varphi}+C_{C \lambda}+C_{R z}+C_{C A}+C_{a x i s}
\end{aligned}
$$

$\mathrm{r}, \lambda$ and $\varphi$ being the measured distance, vertical angle and horizontal angle.

The corrections that come into play in the model are $C_{\text {signal }}, C_{C \lambda}, C_{V \varphi}, C_{R x}, C_{R y}, C_{R z}, C_{a x i s}, C_{C A}$ corresponding to the corrections due to the scanned signal, to the vertical circle compensator, the lack of verticality of the main axis, the resolution of the indicator device at the $x, y, z$ coordinates, the lack of perpendicularity between the axes of the instrument [5], and the correction in the measure of the distance due to atmospheric conditions which include the correction due to the temperature, $\mathrm{C}_{\mathrm{T}}$, the one due to the pressure, $\mathrm{C}_{\mathrm{P}}$, and to the humidity, $\mathrm{C}_{\mathrm{H}}$.

Some of the above-mentioned corrections are unknown, others may be considered null although this fact does not imply that they are exempt from uncertainty. It should be pointed out that in this research many values of the associated uncertainties will be Type B ones since it is not possible to reproduce the repeatability conditions for every scanned point; hence there is no room here for statistical analysis.

\subsection{Combined standard uncertainty}

Two different methodologies are used to estimate the combined standard uncertainty: one of them applies the law of propagation of uncertainty taken in by the GUM [2] and the other calculates the standard measurement 
uncertainties by means of the Monte Carlo method through the simulation of random variables [6].

There is no information about the contributions to the uncertainties $u\left(C_{R}\right), u\left(C_{C A}\right), u\left(C_{\text {axis }}\right)$ in the technical specifications of the TLS equipment models employed. These contributions are considered to be included into the experimental standard deviations $s_{x}, s_{y}$ and $s_{z}$ of each scanned point; this would give an idea of the repeatability of the measurement.

The differences between the estimated standard uncertainties of the scanned points in each series for every spherical signal with the same instrument are in the order of micrometers.

For each explored series, $\mathrm{n}$ being the number of explored points, we have:

$$
\begin{aligned}
& \bar{u}_{x p o \text { int } s}=\frac{\sum_{i=1}^{n} u_{p o \text { int } s}}{n} \\
& \bar{u}_{y p \text { int } s}=\frac{\sum_{i=1}^{n} u_{p o \text { int } s}}{n} \\
& \bar{u}_{z \text { points }}=\frac{\sum_{i=1}^{n} u_{p o \text { int } s}}{n}
\end{aligned}
$$

Since the number of series or scans carried out is five, we obtain:

$$
\begin{aligned}
& \bar{u}_{x}=\frac{\sum_{i=1}^{5} \bar{u}_{x p o \text { int } s}}{5} \\
& \bar{u}_{y}=\frac{\sum_{i=1}^{5} \bar{u}_{y p o \text { int } s}}{5} \\
& \bar{u}_{z}=\frac{\sum_{i=1}^{5} \bar{u}_{z p o \text { int } s}}{5}
\end{aligned}
$$

The Monte Carlo method is a practical alternative to the GUM's approach on uncertainty; its purpose is getting an approximate numerical representation of a function. The input variables have associated uncertainties of which the probability distribution is known in a rough way; a procedure is developed by using Matlab which carries out $10^{6}$ iterations for every value of the associated uncertainties coming into play in the function; this provides approximately $95 \%$ of coverage interval for the output magnitude, such that the amplitude of the interval is correct with one or two significant decimal figures. It has been possible to verify that the results obtained with the Monte Carlo method are practically coincident with the results obtained by applying the law of propagation of uncertainty.

\subsection{Estimation of the expanded uncertainty}

The expanded uncertainty, $U$, is obtained by applying to the combined standard uncertainty $u_{c}(y)$ a coverage factor $k$, i.e. $U=k u_{c}(y)$. The selected coverage factor is $k=2$ which defines an interval with a level of confidence of approximately $95 \%$.

Tables 1 and 2 below show the expanded

\begin{tabular}{|c|c|c|c|c|}
\hline \multirow[b]{2}{*}{ Equipment } & \multirow[b]{2}{*}{ Target } & \multicolumn{3}{|c|}{$\begin{array}{l}\text { Field } \\
\text { (distance TLS-Sphere } \sim 15-20 \mathrm{~m} \text { ) }\end{array}$} \\
\hline & & $\begin{array}{l}\bar{u}_{x} \\
(\mathbf{m m})(k=2)\end{array}$ & $\begin{array}{l}\bar{u}_{y} \\
(\mathbf{m m})(k=2)\end{array}$ & $\begin{array}{l}\bar{u}_{z} \\
(\mathbf{m m})(k=2)\end{array}$ \\
\hline \multirow{3}{*}{$\begin{array}{l}\text { Trimble GX } \\
\text { 3D scanner }\end{array}$} & $\mathrm{A}$ & 2,5930 & 2,5994 & 2,7706 \\
\hline & B & 2,3722 & 2,3538 & 2,1306 \\
\hline & $\mathrm{C}$ & 2,3626 & 2,3660 & 2,1320 \\
\hline \multirow{3}{*}{$\begin{array}{l}\text { Leica Scan } \\
\text { Station C10 }\end{array}$} & $\mathrm{A}$ & 6,0564 & 5,6572 & 2,3550 \\
\hline & B & 1,8978 & 7,9760 & 1,8258 \\
\hline & $\mathrm{C}$ & 7,6110 & 3,8862 & 3,0070 \\
\hline \multirow{3}{*}{$\begin{array}{l}\text { Leica HDS } \\
6200\end{array}$} & A & 2,8482 & 2,8400 & 0,4386 \\
\hline & $\mathrm{B}$ & 2,8680 & 2,8082 & 0,3806 \\
\hline & $\mathrm{C}$ & 2,8400 & 2,8852 & 0,6266 \\
\hline
\end{tabular}
uncertainties of the $3 \mathrm{D}$ coordinates of the points in the spherical signals obtained with the TLS equipment models considered in this study both in field and lab conditions.

Table 1. In-field standard expanded uncertainty

Table 2. In-lab standard expanded uncertainty

\begin{tabular}{lllll}
\hline & \multicolumn{5}{c}{$\begin{array}{l}\text { Laboratory } \\
(\text { distance }\end{array}$} \\
\cline { 3 - 5 } Equipment & Target & $\bar{u}_{x}$ & $\bar{u}_{y}$ & $\bar{u}_{z}$ \\
& & $(\mathbf{m m})(\boldsymbol{k}=\mathbf{2})$ & $\mathbf{( m m})(\boldsymbol{k}=\mathbf{2})$ & $(\mathbf{m m})(\boldsymbol{k}=\mathbf{2})$ \\
\hline \multirow{2}{*}{ Trimble GX } & $\mathrm{A}$ & 1,3160 & 2,4974 & 0,4306 \\
3D scanner & $\mathrm{B}$ & 2,6580 & 0,8590 & 0,5386 \\
& $\mathrm{C}$ & 2,7940 & 0,4570 & 0,5220 \\
Leica Scan & $\mathrm{A}$ & 5,6680 & 5,6446 & 0,3696 \\
Station C10 & $\mathrm{B}$ & 5,5500 & 5,5764 & 0,4620 \\
& $\mathrm{C}$ & 5,6596 & 5,6678 & 0,4478 \\
Leica HDS & $\mathrm{A}$ & 2,8276 & 2,8204 & 0,0820 \\
6200 & $\mathrm{B}$ & 2,7942 & 2,8028 & 0,0950 \\
& $\mathrm{C}$ & 2,8246 & 2,8288 & 0,0936 \\
\hline
\end{tabular}

\section{Degree of concordance between the measurements of a single measurand through surface models}

In order to know the behaviour of the equipment types through the repeatability and reproducibility in the measure of a measurand, the corresponding surface models have been generated based on the point clouds created by the different TLS equipment models.

The results show that the repeatability values are submillimetric and the variation between the sphere explorations is minimal, except for the HDS 6200 model in field conditions which could be due to the type of equipment measurement, the lighting of the spherical signals, their position, etc. Regarding reproducibility, the analysis carried out allows detecting whether there are significant differences between the results when performing scans in different measurement conditions.

The following points are taken into account in the analysis:

. Definition of the model: a hypothetical model of distribution of probability of the observations is determined. In this case, the model includes the basic 
hypotheses of normalcy, homoscedasticity and independence.

. Estimation: the unknown parameters are estimated, in our case the diameter averages and variances of the normal distributions, always using the sample information.

Diagnosis: the basic hypotheses defined in the model are compared and contrasted.

Contrasting average equality, the $\mathrm{H}_{0}$ rejection indicates that the procedures bring about a significantly different effect on the averages. On the contrary, the lack of $\mathrm{H}_{0}$ rejection indicates that the difference between the averages is not big enough for it to be detected with the given sampling size. For this reason the lack of $\mathrm{H}_{0}$ rejection does not necessarily mean that it is accepted, therefore to imply that the average equality is accepted may be misleading. In both cases, the result is given with a $95 \%$ level of confidence. Contrasting variance equality, the acceptance of the null hypothesis implies that the variances of each dataset may be considered equal. See Table 3.

Table 3.Variance analysis

\begin{tabular}{|c|c|c|c|c|}
\hline \multicolumn{5}{|c|}{ In-field vs laboratory conditions } \\
\hline $\begin{array}{l}\text { Equipment } \\
\text { model }\end{array}$ & Signal & $\begin{array}{l}\text { Contrasting } \\
\text { average } \\
\text { equality, } \mathrm{H}_{0} \\
\text { rejection? }\end{array}$ & $\begin{array}{l}\text { Interval of } \\
\text { Confidence } \\
\mu_{1}-\mu_{2}(\mathrm{~mm})\end{array}$ & $\begin{array}{l}\text { Contrasting } \\
\text { variance } \\
\text { equality, } \mathrm{H}_{0} \\
\text { rejection? }\end{array}$ \\
\hline \multirow{3}{*}{$\begin{array}{l}\text { Trimble GX } \\
\text { 3D scanner }\end{array}$} & A & Yes & $2,209 \pm 0,771$ & No \\
\hline & B & Yes & $1,479 \pm 0,609$ & No \\
\hline & $\mathrm{C}$ & Yes & $3,512 \pm 0,157$ & No \\
\hline \multirow{3}{*}{$\begin{array}{l}\text { Leica Scan } \\
\text { Station C10 }\end{array}$} & A & Yes & $-1,468 \pm 0,501$ & No \\
\hline & B & Yes & $-1,048 \pm 0,265$ & No \\
\hline & $\mathrm{C}$ & Yes & $-1,308 \pm 0,298$ & No \\
\hline \multirow{3}{*}{$\begin{array}{l}\text { Leica HDS } \\
6200\end{array}$} & A & No & ------- & Yes \\
\hline & B & Yes & $-3,208 \pm 0,746$ & No \\
\hline & $\mathrm{C}$ & Yes & $-3,312 \pm 3,615$ & Yes \\
\hline
\end{tabular}

On the other hand, in the contrast of variance equality, $\mathrm{H}_{0}$ is not rejected in any of the cases in which an equipment model based on the principle of flight time measurement is used (Trimble GX 3D scanner and Leica Scan Station C10). It is confirmed that the equipment models reproduce measures whose scattering may be considered similar in field or lab conditions, i.e. the instrument behaves similarly as far as measure repeatability is concerned. However, this is just one contribution to measurement uncertainty and it does not mean that the conditions of the observation do not affect the measures taken.

Table 4.Variance analysis with the Trimble GX 3D scanner and Leica Scan Station C10 equipment models

\begin{tabular}{|ccccc|}
\hline \multicolumn{4}{|c|}{ Trimble GX 3D scanner $\boldsymbol{v s}$ Leica Scan Station C10 } \\
\hline $\begin{array}{c}\text { Conditions } \\
\text { of } \\
\text { observation }\end{array}$ & Signal & $\begin{array}{c}\text { Contrasting } \\
\text { average } \\
\text { equality, } \mathbf{H}_{\mathbf{0}} \\
\text { rejection? }\end{array}$ & $\begin{array}{c}\text { Interval of } \\
\text { Confidence } \\
\boldsymbol{\mu}_{\mathbf{1}}-\boldsymbol{\mu}_{\mathbf{2}} \mathbf{( m m )}\end{array}$ & $\begin{array}{c}\text { Contrasting } \\
\text { variance } \\
\text { equality, } \mathbf{H}_{\mathbf{0}} \\
\text { rejection? }\end{array}$ \\
\hline \multirow{2}{*}{ Field } & $\mathrm{A}$ & Yes & $1,021 \pm 0,967$ & No \\
& $\mathrm{B}$ & Yes & $2,850 \pm 0,442$ & No \\
& $\mathrm{C}$ & Yes & $4,668 \pm 0,237$ & No \\
\hline \multirow{2}{*}{ Laboratory } & $\mathrm{A}$ & Yes & $0,330 \pm 0,313$ & Yes \\
& $\mathrm{B}$ & Yes & $0,323 \pm 0,496$ & No \\
& $\mathrm{C}$ & Yes & $-0,152 \pm 2,69$ & No \\
\hline
\end{tabular}

Table 4 shows the results obtained in the variance analysis, contrasting the results of both equipment models, Trimble GX 3D scanner and Leica Scan Station $\mathrm{C} 10$ that are based on the same measurement principle, namely flight time; they have been used under the same working conditions.

\section{Conclusions}

On the basis of the analysis of the numerical results obtained with real data, the following conclusions may be drawn:

. Lackof standardization was detected in the indication of the uncertainties provided by the manufacturers; this hindered interpretation of the technical specifications of the equipment models since clearly defined values were not available.

- Since the same procedure was developed and applied with the three different TLS equipment models, it is possible to make a relative comparison among the results obtained, hence to facilitate the choice of equipment best fitting the needs of a specific project.

. The variance analysis is essential since it reveals interesting conclusions regarding TLS equipment behaviour.

. Concerning reproducibility analysis, it is advisable to work in different conditions of observation and/or to employ equipment models of different manufacturers based on the same measurement principle.

. The geometric model of the selected signals has allowed treatment of the data with great reliability. A research study of measure reproducibility as a function of texture and colour of the material object of exploration is a remaining issue.

\section{References}

1. I. Greenway, XXIII FIG International Congress, Munich, Germany, October, Standards-their vital role, (2006)

2. BIPM, IEC, IFCC, ILAC, ISO, IUPAC, IUPAP and OIML. JCGM 100:2008 (GUM 1995 with minor corrections), Evaluation of Measurement Data-Guide to the Expression of Uncertainty in Measurement, (2008)

3. R. Staiger, International Federation of Surveyors. Article of the Month, June, Push the Button - or Does the Art of Measurement Still Exist?, (2009)

4. R. Staiger, FIG Working Week 2005 and GSDI-8 Cairo, Egypt. April 16-21, Geometrical Quality of Laser Scans, (2005)

5. J. M. Rüeger, R. Gottwald, Trans Tasman Surveyor No. 4, Field Tests and Checks for Electronic Tacheometers, 18 - 26, (2001)

6. Joint Committee for Guides in Metrology 2008. JCGM 101:2008. Evaluation of measurement data. Supplement 1 to the "Guide to the expression of uncertainty in measurement". Propagation of distributions using a Monte Carlo method, (2008) 